\title{
A Note on the Structure of Affine Subspaces of $L^{2}\left(\mathbb{R}^{d}\right)$
}

\author{
Fengying Zhou, Xiaoyong Xu \\ School of Science, East China Institute of Technology, Nanchang, China \\ Email: zhoufengying@ecit.cn, xxy@ecit.cn
}

Received 8 January 2015; accepted 26 January 2015; published 28 January 2015

Copyright (C) 2015 by authors and Scientific Research Publishing Inc.

This work is licensed under the Creative Commons Attribution International License (CC BY).

http://creativecommons.org/licenses/by/4.0/

(c) () Open Access

\section{Abstract}

This paper investigates the structure of general affine subspaces of $L^{2}\left(\mathbb{R}^{d}\right)$. For a $d \times d$ expansive matrix $A$, it shows that every affine subspace can be decomposed as an orthogonal sum of spaces each of which is generated by dilating some shift invariant space in this affine subspace, and every non-zero and non-reducing affine subspace is the orthogonal direct sum of a reducing subspace and a purely non-reducing subspace, and every affine subspace is the orthogonal direct sum of at most three purely non-reducing subspaces when $|\operatorname{det} A|=2$.

\section{Keywords}

\section{Affine Subspace, Reducing Subspace, Shift Invariant Subspace, Orthogonal Sum}

\section{Introduction}

Let $A$ be a $d \times d$ expansive matrix. Define the dilation operator $D$ and the shift operator $T_{k}, k \in \mathbb{Z}^{d}$, by

$$
D f(\cdot)=|\operatorname{det} A|^{\frac{1}{2}} f(A \cdot) \text { and } T_{k} f(\cdot)=f(\cdot-k), \quad f \in L^{2}\left(\mathbb{R}^{d}\right),
$$

respectively. It is easy to check that they are both unitary operators on $L^{2}\left(\mathbb{R}^{d}\right)$. Given a closed subspace $X$ of $L^{2}\left(\mathbb{R}^{d}\right), X$ is called a shift invariant subspace if $T_{k} X=X$ for every $k \in \mathbb{Z}^{d} ; X$ is called a reducing subspace of $L^{2}\left(\mathbb{R}^{d}\right)$ if $D X=X$ and $T_{k} X=X$ for every $k \in \mathbb{Z}^{d} ; X$ is called an affine subspace of $L^{2}\left(\mathbb{R}^{d}\right)$ if there exists an at most countable subset $\Phi$ of $L^{2}\left(\mathbb{R}^{d}\right)$ such that

$$
X=\overline{\operatorname{span}}\left\{D^{j} T_{k} \phi: \phi \in \Phi, j \in \mathbb{Z}, k \in \mathbb{Z}^{d}\right\} .
$$


In this case, we say that $\Phi$ generates the affine subspace $X$. An affine subspace, which does not contain any non-zero reducing subspace, is called purely non-reducing. By Theorem 3.1 in [1], a closed subspace $X$ of $L^{2}\left(\mathbb{R}^{d}\right)$ is an affine subspace if and only if $X=\overline{\operatorname{span}}\left\{D^{j} f: f \in M, j \in \mathbb{Z}\right\}$ for some shift invariant subspace $M$. Therefore an affine subspace $X$ of $L^{2}\left(\mathbb{R}^{d}\right)$ is a reducing subspace if and only if it is shift invariant. So far, the study of reducing subspaces has achieved fruitful results. The existence and construction of wavelet frames for an arbitrary reducing subspace can be seen in [2]-[7]. For one-dimensional case $A=2$, Gu and Han investigated the existence of Parseval wavelet frames for singly generated affine subspaces in [8] and the structural properties of affine subspaces in [9]. For a given $d \times d$ expansive matrix A, Zhou and Li studied the construction of wavelet frames in the setting of finitely generated affine subspaces of $L^{2}\left(\mathbb{R}^{d}\right)$ in [10]. For a general $d \times d$ expansive matrix $A$, this paper focuses on the structure of affine subspaces of $L^{2}\left(\mathbb{R}^{d}\right)$, which is a continuation of the literature [10] and has not been investigated yet.

\section{Main Results}

Lemma 1. Let $X$ and $Y$ be closed subspaces of a Hilbert space $H$ and $P_{X^{\perp}}$ be the orthogonal projection onto $X^{\perp}$. Then
1) $(X \cap Y)^{\perp}=\overline{\operatorname{span}}\left\{X^{\perp}, Y^{\perp}\right\}$;
2) $\overline{P_{X^{\perp}} Y}=(\overline{\operatorname{span}}\{X, Y\}) \ominus X$.

Proof. 1) Obviously, $(X \cap Y)^{\perp}=X^{\perp} \cup Y^{\perp} \subset \overline{\operatorname{span}}\left\{X^{\perp}, Y^{\perp}\right\}$. For the other direction, note that

$$
X^{\perp} \subset \overline{\operatorname{span}}\left\{X^{\perp}, Y^{\perp}\right\} \text { and } Y^{\perp} \subset \overline{\operatorname{span}}\left\{X^{\perp}, Y^{\perp}\right\},
$$

then

$$
\left(\overline{\operatorname{span}}\left\{X^{\perp}, Y^{\perp}\right\}\right)^{\perp} \subset X \text { and }\left(\overline{\operatorname{span}}\left\{X^{\perp}, Y^{\perp}\right\}\right)^{\perp} \subset Y .
$$

So $\left(\overline{\operatorname{span}}\left\{X^{\perp}, Y^{\perp}\right\}\right)^{\perp} \subset X \cap Y$. Therefore, $(X \cap Y)^{\perp} \subset \overline{\operatorname{span}}\left\{X^{\perp}, Y^{\perp}\right\}$. Thus 1) holds.

2) For $f \in P_{X^{\perp}} Y \subset P_{X^{\perp}} \overline{\operatorname{span}}\{X, Y\}$, there exists some $g \in \overline{\operatorname{span}}\{X, Y\}$ such that $f=P_{X^{\perp}} g$. So $g=P_{X^{\perp}} g+P_{X} g=f+P_{X} g$, or $f=g-P_{X} g \in \overline{\operatorname{span}}\{X, Y\}$, which shows $f \in \overline{\operatorname{span}}\{X, Y\} \ominus X$ due to the fact that $f \in X^{\perp}$. For $f \in \overline{\operatorname{span}}\{X, Y\} \ominus X$, we have $f \in \overline{\operatorname{span}}\{X, Y\}$ and $f \perp X$. Thus for any $\epsilon>0$, there is $g$ with $\|g\|<\epsilon$ and $h_{1} \in X$ and $h_{2} \in Y$ such that $f=h_{1}+h_{2}+g$. Consequently,

$$
f=P_{X^{\perp}} f=P_{X^{\perp}}\left(h_{1}+h_{2}+g\right)=P_{X^{\perp}} g+P_{X^{\perp}} h_{2} \in \overline{P_{X^{\perp}} Y}
$$

since $\left\|P_{X^{\perp}} g\right\| \leq\|g\|<\epsilon$. The proof is completed.

Lemma 2. Let $\left\{X_{n}: n \in \mathbb{Z}\right\}$ be a monotone sequence of subspaces in a Hilbert space $\mathcal{H}$.

1) If $\left\{X_{n}: n \in \mathbb{Z}\right\}$ is increasing, then

$$
\overline{\operatorname{span}}\left\{X_{n}: n \in \mathbb{Z}\right\}=\overline{\bigcup_{n \in \mathbb{Z}} X_{n}}=\left(\bigcap_{n \in \mathbb{Z}} X_{n}\right) \oplus\left(\bigoplus_{n \in \mathbb{Z}}\left(X_{n+1} \ominus X_{n}\right)\right) .
$$

2) If $\left\{X_{n}: n \in \mathbb{Z}\right\}$ is decreasing, then

$$
\overline{\operatorname{span}}\left\{X_{n}: n \in \mathbb{Z}\right\}=\overline{\bigcup_{n \in \mathbb{Z}} X_{n}}=\left(\bigcap_{n \in \mathbb{Z}} X_{n}\right) \oplus\left(\bigoplus_{n \in \mathbb{Z}}\left(X_{n} \ominus X_{n+1}\right)\right) .
$$

Proof. We only prove 1) since 2) can be obtained similarly. Since $\left\{X_{n}: n \in \mathbb{Z}\right\}$ is increasing, the first equality is obvious and

$$
\left(\bigcap_{n \in \mathbb{Z}} X_{n}\right) \oplus\left(\bigoplus_{n \in \mathbb{Z}}\left(X_{n+1} \odot X_{n}\right)\right) \subset \overline{\bigcup_{n \in \mathbb{Z}} X_{n}}
$$


If $f \in \overline{\bigcup_{n \in \mathbb{Z}} X_{n}}$, then for any $\epsilon>0$, there exists $g \in \mathcal{H}, n_{0} \in \mathbb{Z}$ and $h \in X_{n_{0}}$ such that $\|g\|<\epsilon$ and $f=g+h$. For such $h$, there is a unique sequence $\left\{h_{n}\right\}_{n=-\infty}^{n_{0}}$ and a unique $\tilde{h}$ such that $h_{n} \in X_{n} \ominus X_{n-1}$ for each $n \leq n_{0}, \quad \tilde{h} \in \bigcap_{n \in \mathbb{Z}} X_{n}$ and $h=\tilde{h}+\sum_{n=-\infty}^{n_{0}} h_{n}$. This means that

$$
f \in \overline{\left(\bigcap_{n \in \mathbb{Z}} X_{n}\right) \oplus\left(\bigoplus_{n \in \mathbb{Z}}\left(X_{n+1} \ominus X_{n}\right)\right)}=\left(\bigcap_{n \in \mathbb{Z}} X_{n}\right) \oplus\left(\bigoplus_{n \in \mathbb{Z}}\left(X_{n+1} \ominus X_{n}\right)\right) .
$$

The proof is completed.

Proposition 1. Suppose that $X$ is an affine subspace of $L^{2}\left(\mathbb{R}^{d}\right)$ with $M$ being its generating shift invariant subspace. Then there exist a shift invariant subspace $M_{1}$ in $X$ and a reducing subspace $Y$ of $L^{2}\left(\mathbb{R}^{d}\right)$ contained in $X$ such that the length of $M_{1}$ is no more than that of $M$ and $X=\left(\underset{n \in \mathbb{Z}}{\oplus} M_{1}\right) \oplus Y$.

Proof. For each $j \in \mathbb{Z}$, define

$$
Y_{j}=\overline{\operatorname{span}}\left\{D^{n} M: n \in \mathbb{Z}, n>j\right\} .
$$

Obviously, $Y_{j+1} \subset Y_{j}$ for $j \in \mathbb{Z}$ and $X=\overline{\bigcup_{j \in \mathbb{Z}} Y_{j}}$. Let $Y=\bigcap_{j \in \mathbb{Z}} Y_{j}$. Similarly to the proof of Proposition 2.2 in [10], we know that $Y$ is a reducing subspace. Now define $M_{1}=Y_{-1} \ominus Y_{0}$. Then $Y_{j} \ominus Y_{j+1}=D^{j+1} M_{1}$ by Lemma 2 and

$$
X=\overline{\bigcup_{j \in \mathbb{Z}} Y_{j}}=\left(\bigcap_{j \in \mathbb{Z}} Y_{j}\right) \oplus\left(\bigoplus_{j \in \mathbb{Z}}\left(Y_{j} \ominus Y_{j+1}\right)\right)=Y \oplus\left(\bigoplus_{j \in \mathbb{Z}} D^{j+1} M_{1}\right)=Y \oplus\left(\bigoplus_{j \in \mathbb{Z}} D^{j} M_{1}\right) .
$$

Suppose that for some subset $\Psi \subset L^{2}\left(\mathbb{R}^{d}\right)$ such that

$$
M=\overline{\operatorname{span}}\left\{T_{k} \psi: k \in \mathbb{Z}^{d}, \psi \in \Psi\right\} \text {. }
$$

Since $Y_{0}$ is a shift invariant subspace, so is $Y_{0}^{\perp}$. Thus for each $k \in \mathbb{Z}^{d}, T_{k} P_{Y_{0}^{\perp}}=P_{Y_{0}^{\perp}} T_{k}$. Also note that $Y_{-1}=\overline{\operatorname{span}}\left\{D^{j} M, M: j \in \mathbb{Z}, j>0\right\}=\overline{\operatorname{span}}\left\{Y_{0}, M\right\}$. Therefore, by Lemma 1,

$$
\begin{aligned}
M_{1} & =Y_{-1} \ominus Y_{0}=\left(\overline{\operatorname{span}}\left\{M, Y_{0}\right\}\right) \ominus Y_{0} \\
& =\overline{P_{Y_{0}^{\perp}} M}=\overline{\operatorname{span}}\left\{P_{Y_{0}^{\perp}} T_{k} \psi: k \in \mathbb{Z}^{d}, \psi \in \Psi\right\} \\
& =\overline{\operatorname{span}}\left\{T_{k} P_{Y_{0}^{\perp}} \psi: k \in \mathbb{Z}^{d}, \psi \in \Psi\right\},
\end{aligned}
$$

which shows that $M_{1}$ is a shift invariant subspace of length no more than the length of $M$. The proof is completed.

Proposition 2. Suppose that $X$ is a non-zero affine subspace of $L^{2}\left(\mathbb{R}^{d}\right)$ and $Q$ is the maximal shift invariant subspace contained in $X$. Then the following hold:

1) $D Q \subset Q$ and $Q \odot D Q$ is a shift invariant subspace contained in $X$;

2) $X=\bigoplus_{n \in Z} D(Q \ominus D Q)$ if and only if $X$ is purely non-reducing subspace of $L^{2}\left(\mathbb{R}^{d}\right)$.

Proof. 1): Obviously, $D Q \subset X$ is shift invariant space since $Q$ is shift invariant. So $D Q \subset Q$ due to the fact that $Q$ is the maximal shift invariant subspace contained in $X$. Thus $Q \ominus D Q$ is a shift invariant subspace contained in $X$.

2): By 1) and Lemma 2, it follows that

$$
X=\overline{\operatorname{span}}\left\{D^{n} Q: n \in \mathbb{Z}\right\}=\overline{\bigcup_{n \in \mathbb{Z}} D^{n} Q}=\left(\bigoplus_{n \in \mathbb{Z}}(Q \ominus D Q)\right) \oplus\left(\bigcap_{n \in \mathbb{Z}} D^{n} Q\right) .
$$


If $X$ is purely non-reducing, then $\bigcap_{n \in \mathbb{Z}} D^{n} Q=\{0\}$ since $\bigcap_{n \in \mathbb{Z}} D^{n} Q=\bigcap_{n \in \mathbb{N}} D^{n} Q$ is a reducing subspace. So $X=\bigoplus_{n \in \mathbb{Z}} D(Q \ominus D Q)$. Suppose $X=\bigoplus_{n \in \mathbb{Z}} D(Q \ominus D Q)$ and $X$ contains a reducing subspace $Y$. Next we only need to show $Y=\{0\}$. Since $Y$ is reducing, we have $Y \subset Q$ and $D Y=Y \subset D Q$, i.e., $Y \subset Q \cap D Q$. Also note that $Q=(Q \odot D Q) \oplus(Q \cap D Q)$. Hence $Y \perp(Q \ominus D Q)$. Thus for each $n \in \mathbb{Z}, \quad Y=D^{n} Y \perp D^{n}(Q \cap D Q)$. Therefore $Y \perp X$, which shows that $Y=\{0\}$. The proof is completed.

Proposition 3. Let $X$ be an affine subspace of $L^{2}\left(\mathbb{R}^{d}\right)$, and define $L:=\overline{\operatorname{span}}\left\{T_{k} X: k \in \mathbb{Z}^{d}\right\} \ominus X$. The $X \cap\left(\overline{\operatorname{span}}\left\{T_{k} L: k \in \mathbb{Z}^{d}\right\}\right)^{\perp}$ is the maximal shift invariant subspace contained in $X$.

Proof. We first show that $X \cap\left(\overline{\operatorname{span}}\left\{T_{k} L: k \in \mathbb{Z}^{d}\right\}\right)^{\perp}$ is shift invariant. For $k \in \mathbb{Z}^{d}$ and $f \in X \cap\left(\overline{\operatorname{span}}\left\{T_{l} L: l \in \mathbb{Z}^{d}\right\}\right)^{\perp}$, it follows that

$$
f \in X, T_{k} f \perp \overline{\operatorname{span}}\left\{T_{l} L: l \in \mathbb{Z}^{d}\right\} .
$$

Next we will show that $T_{k} f \in X$ by contradiction. If there exists some $k_{0} \in \mathbb{Z}^{d}$ such that $T_{k_{0}} f \notin X$, then

$$
T_{k_{0}} f=\eta+\zeta \text { for } \eta \in X \text { and } 0 \neq \zeta \in X^{\perp} .
$$

So $\zeta=T_{k_{0}} f-\eta \in \overline{\operatorname{span}}\left\{T_{l} X: l \in Z^{d}\right\} \ominus X=L$. Therefore $T_{-k_{0}} \zeta \in \overline{\operatorname{span}}\left\{T_{l} L: l \in \mathbb{Z}^{d}\right\}$, which implies that $T_{-k_{0}} \zeta \perp f$ since $f \in\left(\overline{\operatorname{span}}\left\{T_{k} L: k \in \mathbb{Z}^{d}\right\}\right)^{\perp}$. Consequently, $\|f\|^{2}=\left\langle f, T_{-k_{0}} \eta+T_{-k_{0}} \zeta\right\rangle=\left\langle f, T_{-k_{0}} \eta\right\rangle \leq\|f\|\|\eta\|$. This leads to a contradiction since $\|f\|>\|\eta\|$. Assume that $M$ is a shift invariant subspace contained in $X$. Obviously $M \perp \overline{\operatorname{span}}\left\{T_{l} X: l \in \mathbb{Z}^{d}\right\} \ominus X=L$. Thus, $M \perp \overline{\operatorname{span}}\left\{T_{l} L: l \in \mathbb{Z}^{d}\right\}$. So $M \subset X \cap\left(\overline{\operatorname{span}}\left\{T_{l} L: l \in \mathbb{Z}^{d}\right\}\right)^{\perp}$. The result follows. The proof is completed.

Lemma 3. Let $X$ and $Y$ be affine subspaces of $L^{2}\left(\mathbb{R}^{d}\right)$ with $X \perp Y$. Let $M$ and $N$ be generating shift invariant subspaces for $X$ and $Y$ respectively. Then $X \oplus Y$ is an affine subspace of $L^{2}\left(\mathbb{R}^{d}\right)$ with $M \oplus N$ as a generating shift invariant subspace.

Proof. Since $X=\overline{\operatorname{span}}\left\{D^{j} M: j \in \mathbb{Z}\right\}$ and $Y=\overline{\operatorname{span}}\left\{D^{j} N: j \in \mathbb{Z}\right\}$, it follows that

$$
\overline{\operatorname{span}}\left\{D^{j}(M \oplus N): j \in \mathbb{Z}\right\}=\overline{\operatorname{span}}\left\{D^{j} M: j \in \mathbb{Z}\right\} \oplus \overline{\operatorname{span}}\left\{D^{j} N: j \in \mathbb{Z}\right\}=X \oplus Y .
$$

The proof is completed.

Lemma 4. Assume $\left\{X_{n}: n \in \mathbb{Z}\right\}$ is a monotone sequence of subspaces in a Hilbert space $\mathcal{H}$ and give a subspace $Y \subset \mathcal{H}$ satisfying $X_{n} \subset Y$ for each $n \in \mathbb{Z}$. Then

$$
\overline{\operatorname{span}}\left\{Y \ominus X_{n}: n \in \mathbb{Z}\right\}=\overline{\bigcup_{n \in \mathbb{Z}} Y \ominus X_{n}}=Y \odot\left(\bigcap_{n \in \mathbb{Z}} X_{n}\right) .
$$

Proof. Since $\left\{X_{n}: n \in \mathbb{Z}\right\}$ is a monotone sequence of subspaces and $X_{n} \subset Y, n \in \mathbb{Z}$, we have $\left\{Y \ominus X_{n}: n \in \mathbb{Z}\right\}$ is also a monotone sequence. Then the first equality follows by Lemma 2. For $x \in \bigcup_{n \in \mathbb{Z}} Y \ominus X_{n}$, there exists some $n_{0} \in \mathbb{Z}$ such that $x \in Y \ominus X_{k_{0}}$, namely $x \in Y$ and $x \perp X_{k_{0}}$. Then $x \in Y \ominus\left(\bigcap_{n \in \mathbb{Z}} X_{n}\right)$. Thus $\bigcup_{n \in \mathbb{Z}} Y \ominus X_{n} \subset Y \ominus\left(\bigcap_{n \in \mathbb{Z}} X_{n}\right)$. So $\overline{\bigcup_{n \in \mathbb{Z}} Y \ominus X_{n}} \subset Y \ominus\left(\bigcap_{n \in \mathbb{Z}} X_{n}\right)$. For the other direction, without loss of generality, assume that $\left\{X_{n}: n \in \mathbb{Z}\right\}$ is increasing. By Lemma 2, 


$$
Y \ominus\left(\bigcap_{n \in \mathbb{Z}} X_{k}\right)=\left(Y \ominus \overline{\bigcup_{n \in \mathbb{Z}} X_{n}}\right) \oplus\left(\overline{\bigcup_{n \in \mathbb{Z}} X_{n}} \ominus\left(\bigcap_{k \in \mathbb{Z}} X_{k}\right)\right)=Y \ominus \overline{\bigcup_{n \in \mathbb{Z}} X_{n}} \oplus\left(\bigoplus_{k \in \mathbb{Z}}\left(X_{k-1} \ominus X_{k}\right)\right)
$$

which shows that $Y \ominus\left(\bigcap_{n \in \mathbb{Z}} X_{k}\right) \subset \overline{\bigcup_{k \in \mathbb{Z}}\left(Y \ominus X_{k}\right)}$. The proof is completed.

Lemma 5. Let $X$ be an affine subspace of $L^{2}\left(\mathbb{R}^{d}\right)$ and $Q$ be the maximal shift invariant subspace contained in $X$. Define $V:=\overline{\operatorname{span}}\left\{T_{k}(X \ominus Q): k \in \mathbb{Z}^{d}\right\}$. Then the following hold:

1) $D^{j} Q \subset D^{j-1} Q$ and $D^{j} V \subset D^{j+1} V$ for $j \in \mathbb{Z}$;

2) $Q \perp V, V=\{0\}$ if and only if $X$ is a reducing subspace of $L^{2}\left(\mathbb{R}^{d}\right)$;

3) $X \perp \bigcap_{j \in \mathbb{Z}} D^{j} V, \bigcap_{j \in \mathbb{Z}} D^{j} V$ is in any reducing subspace of $L^{2}\left(\mathbb{R}^{d}\right)$ containing $X$.

Proof. 1): Note that we only need to show $D Q \subset Q$ and $D^{-1} V \subset V$. While $D Q \subset Q$ follows by Proposition 2. So $Q \subset D^{-1} Q$. Thus we have

$$
\begin{aligned}
D^{-1} V & =\overline{\operatorname{span}}\left\{D^{-1} T_{k}(X \ominus Q): k \in \mathbb{Z}^{d}\right\}=\overline{\operatorname{span}}\left\{T_{A k}\left(X \ominus D^{-1} Q\right): k \in \mathbb{Z}^{d}\right\} \\
& \subset \overline{\operatorname{span}}\left\{T_{A k}(X \ominus Q): k \in \mathbb{Z}^{d}\right\} \subset \overline{\operatorname{span}}\left\{T_{k}(X \ominus Q): k \in \mathbb{Z}^{d}\right\}=V .
\end{aligned}
$$

2): Since $Q$ is shift invariant and $Q \perp(X \odot Q)$, it follows that

$$
Q \perp \overline{\operatorname{span}}\left\{T_{k}(X \ominus Q): k \in \mathbb{Z}^{d}\right\}=V .
$$

If $X$ is a reducing subspace, then $Q=X$. By the definition of $V$, we have $V=\{0\}$. If $V=\{0\}$, then $X=Q$, which shows that $X$ is shift invariant. Thus $X$ is a reducing subspace.

3): By 1) and 2), we have $D^{j} Q \perp D^{l} V$ for all $j, \quad l \in \mathbb{Z}$ with $j \geq l$. Thus for each $j \in \mathbb{Z}$,

$D^{j} Q \perp \bigcap_{l \leq j} D^{l} V=\bigcap_{l \in \mathbb{Z}} V$. Therefore $X=\overline{\operatorname{span}}\left\{D^{j} Q: j \in \mathbb{Z}\right\} \perp \bigcap_{l \in \mathbb{Z}} V$. Let $M$ be a reducing subspace containing $X$.

Then $V=\overline{\operatorname{span}}\left\{T_{k}(X \odot Q): k \in \mathbb{Z}^{d}\right\} \subset M$. So for each $j \in \mathbb{Z}, D^{j} V \subset D^{j} M=M$. Hence $\bigcap_{j \in \mathbb{Z}} D^{j} V \subset M$.

The proof is completed.

Proposition 4. Let $X$ and $Y$ be affine subspaces of $L^{2}\left(\mathbb{R}^{d}\right)$ satisfying $X \subset Y$. Let $Q$ and $S$ be the maximal shift invariant subspaces contained in $X$ and $Y$ respectively. Define $V:=\overline{\operatorname{span}}\left\{T_{k}(X \ominus Q): k \in \mathbb{Z}^{d}\right\}$. Then $\left(S \cap V^{\perp}\right) \ominus Q$ is the maximal shift invariant subspace contained in $Y \ominus X$.

Proof. Let $M$ be a shift invariant subspace contained in $Y \ominus X$. By Lemma 3 and the maximality of $S$ as a shift invariant subspace in $Y$, we have $M \oplus Q \subset S$. Note that $Q \perp(X \ominus Q)$ and $M \perp X$. Then $(M \oplus Q) \perp(X \ominus Q)$. So $(M \oplus Q) \perp \overline{\operatorname{span}}\left\{T_{k}(X \oplus Q): k \in \mathbb{Z}^{d}\right\}=V$. Hence $(M \oplus Q) \subset\left(S \cap V^{\perp}\right)$. Therefore $M \subset\left(S \cap V^{\perp}\right) \ominus Q$. The proof is completed.

Proposition 5. Let $X$ and $Y$ be affine subspaces of $L^{2}\left(\mathbb{R}^{d}\right)$ satisfying $X \subset Y$. Let $Q$ and $S$ be the maximal shift invariant subspaces contained in $X$ and $Y$ respectively. Define $V:=\overline{\operatorname{span}}\left\{T_{k}(X \ominus Q): k \in \mathbb{Z}^{d}\right\}$. Then $Y \ominus X$ is an affine subspace of $L^{2}\left(\mathbb{R}^{d}\right)$ if and only if $Y=\overline{\operatorname{span}}\left\{D^{l}\left(S \cap V^{\perp}\right): l \in \mathbb{Z}\right\}$.

Proof. According to Proposition 4, $\left(S \cap V^{\perp}\right) \ominus Q$ is the maximal shift invariant subspace in $Y \ominus X$. If $Y \ominus X$ is an affine subspace, then by Lemma $3, S \cap V^{\perp}$ is a generating shift invariant subspace for $Y$, i.e., $Y=\overline{\operatorname{span}}\left\{D^{l}\left(S \cap V^{\perp}\right): l \in Z\right\}$. Now suppose $Y=\overline{\operatorname{span}}\left\{D^{l}\left(S \cap V^{\perp}\right): l \in \mathbb{Z}\right\}$. Since $D^{l+1} S \subset D^{l} S$ and $D^{l} V \subset D^{l+1} V$ by Lemma 5 for $l \in \mathbb{Z}$, we have $D^{l+l}\left(S \cap V^{\perp}\right) \subset D^{l}\left(S \cap V^{\perp}\right)$ for $l \in \mathbb{Z}$. Thus by Lemma 4 ,

$$
Y=\overline{\operatorname{span}}\left\{D^{l}\left(S \cap V^{\perp}\right): l \in \mathbb{Z}\right\}=\overline{\bigcup_{l \in \mathbb{Z}} D^{l}\left(S \cap V^{\perp}\right)}
$$


Write $M:=\left(S \cap V^{\perp}\right) \ominus Q$ and $\tilde{M}:=(Y \ominus X) \ominus\left((Y \ominus X) \cap\left(S \cap V^{\perp}\right)\right)$. Then $\tilde{M} \subset M$. In fact,

$$
\begin{aligned}
(Y \ominus X) \ominus\left(S \cap V^{\perp} \cap Q^{\perp}\right) & =(Y \ominus X) \cap\left(\left(S \cap V^{\perp}\right) \cap Q^{\perp}\right)^{\perp} \\
& =(Y \ominus X) \cap\left(\left(S \cap V^{\perp}\right)^{\perp} \cup Q\right) \\
& =\left((Y \ominus X) \cap\left(S \cap V^{\perp}\right)^{\perp}\right) \cup((Y \ominus X) \cap Q) \\
& =(Y \ominus X) \cap\left(S \cap V^{\perp}\right)^{\perp} .
\end{aligned}
$$

Hence

$$
(Y \ominus X) \ominus\left((Y \ominus X) \cap\left(S \cap V^{\perp}\right)^{\perp}\right) \subset\left(\left(S \cap V^{\perp}\right) \ominus Q\right)
$$

due to the fact that $\mathcal{H} \ominus \mathcal{M} \subset \mathcal{L}$ is equivalent to $\mathcal{H} \odot \mathcal{L} \subset \mathcal{M}$ for a given Hilbert space $\mathcal{H}$ with its two subspaces $\mathcal{L}$ and $\mathcal{M}$. Also by Lemma 4 , we have

$$
\begin{aligned}
& \overline{\operatorname{span}}\left\{D^{l} \tilde{M}: l \in \mathbb{Z}\right\}=\overline{\bigcup_{l \in \mathbb{Z}} D^{l} \tilde{M}} \\
& =(Y \ominus X) \ominus\left((Y \ominus X) \cap \bigcap_{l \in \mathbb{Z}}\left(D^{l}\left(S \cap V^{\perp}\right)\right)^{\perp}\right) \\
& =(Y \ominus X) \ominus\left((Y \ominus X) \cap\left(\overline{\bigcup_{l \in \mathbb{Z}} D^{l}\left(S \cap V^{\perp}\right)}\right)^{\perp}\right) \\
& =(Y \ominus X) \ominus\left((Y \ominus X) \cap Y^{\perp}\right)=Y \ominus X .
\end{aligned}
$$

So $Y \ominus X=\overline{\operatorname{span}}\left\{D^{l} \tilde{M}: l \in \mathbb{Z}\right\}=\overline{\operatorname{span}}\left\{D^{l} M: l \in \mathbb{Z}\right\}$. The proof is completed.

Proposition 6. Let $X$ and $Y$ be two affine subspaces of $L^{2}\left(\mathbb{R}^{d}\right)$ with $X \subset Y$. Then the following holds.

1) $Y \ominus X$ is affine if $X$ is reducing;

2) $\bigcap_{n \in \mathbb{Z}} D^{n} V=\{0\}$ if $Y$ is reducing and $Y \ominus X$ is affine, where $V=\overline{\operatorname{span}}\left\{T_{k}(X \ominus Q): k \in \mathbb{Z}^{d}\right\}$ and $Q$ is the maximal shift invariant subspace in $X$.

Proof. 1): By Lemma 5, $V=\{0\}$ with $X$ being a reducing subspace. Then $S \ominus Q$ is the maximal shift invariant subspace for $Y \ominus X$ by Proposition 4. Now we only need to show that $Y \ominus X=\overline{\operatorname{span}}\left\{D^{j}(S \ominus Q): j \in \mathbb{Z}\right\}$. Note that

$$
(S \ominus Q) \oplus X=\overline{\operatorname{span}}\{(S \ominus Q) \oplus Q, X\}
$$

due to the facts that $(S \ominus Q) \perp X$ and $Q \subset X$. So by Lemma 1,

$$
\overline{P_{X^{\perp}} S}=\overline{\operatorname{span}}\{S, X\} \ominus X=\overline{\operatorname{span}}\{(S \ominus Q) \oplus Q, X\} \ominus X=(S \ominus Q) \oplus X \ominus X=S \ominus Q .
$$

Observe that $P_{X^{\perp}} D^{l}=D^{l} P_{X^{\perp}}$ since $X^{\perp}$ is invariant under $D^{l}$ for $l \in \mathbb{Z}$. Therefore,

$$
\begin{aligned}
& \overline{\operatorname{span}}\left\{D^{j}(S \ominus Q): j \in \mathbb{Z}\right\} \\
& =\overline{\operatorname{span}}\left\{D^{j} \overline{P_{X^{\perp}} S}: j \in \mathbb{Z}\right\}=\overline{\operatorname{span}}\left\{D^{j} P_{X^{\perp}} S: j \in \mathbb{Z}\right\}=\overline{\operatorname{span}}\left\{P_{X^{\perp}} D^{j} S: j \in \mathbb{Z}\right\} \\
& =\overline{P_{X^{\perp}} \overline{\operatorname{span}}\left\{D^{j} S: j \in \mathbb{Z}\right\}}=\overline{P_{X^{\perp}} Y}=\overline{\operatorname{span}}\{X, Y\} \odot X=Y \ominus X .
\end{aligned}
$$

2): According to Proposition 5, it follows that $Y=\overline{\bigcup_{l \in \mathbb{Z}} D^{l}\left(Y \cap V^{\perp}\right)}$. By Lemma 4, 


$$
\begin{aligned}
Y & =\overline{\bigcup_{l \in \mathbb{Z}} D^{l}\left(Y \cap V^{\perp}\right)}=\overline{\bigcup_{l \in \mathbb{Z}}\left(Y \cap\left(D^{l} V^{\perp}\right)\right)}=\overline{Y \cap\left(\bigcup_{l \in \mathbb{Z}}\left(D^{l} V^{\perp}\right)\right)} \\
& \subset Y \cap \overline{\bigcup_{l \in \mathbb{Z}}\left(D^{l} V^{\perp}\right)}=Y \cap\left(\bigcap\left(D^{l} V\right)\right)^{\perp},
\end{aligned}
$$

which shows that $Y \subset\left(\bigcap_{l \in \mathbb{Z}}\left(D^{l} V\right)\right)^{\perp}$, i.e., $\bigcap_{l \in \mathbb{Z}}\left(D^{l} V\right) \subset Y^{\perp}$. Since $\bigcap_{l \in \mathbb{Z}}\left(D^{l} V\right)$ is contained in any reducing space containing $X$ by Lemma 4, $\bigcap_{l \in \mathbb{Z}}\left(D^{l} V\right) \subset Y$. Consequently $\bigcap_{l \in \mathbb{Z}}\left(D^{l} V\right)=\{0\}$. The proof is completed.

Theorem 7. Let $X$ be an affine subspace of $L^{2}\left(\mathbb{R}^{d}\right)$. Then the following holds.

1) There exist a shift invariant subspace $M$ in $X$ such that $D^{n} M \perp D^{m} M$ for $n, m \in \mathbb{Z}$ with $n \neq m$, and $X=\bigoplus_{n \in \mathbb{Z}} D^{n} M$

2) If $X$ is a non-zero reducing subspace and $|\operatorname{det} A|=2$, then there exist two purely non-reducing affine subspaces $X_{1}$ and $X_{2}$ such that $X=X_{1} \oplus X_{2}$;

3) If $X$ is non-zero and not reducing, then there exists a unique decomposition $X=X_{1} \oplus X_{2}$ with $X_{1}$ be reducing and $X_{2}$ being purely non-reducing;

4) If $X$ is non-zero and $|\operatorname{det} A|=2$, then $X$ is the orthogonal direct sum of at most three purely non-reducing affine subspace.

Proof. 1): By Proposition 1, it follows that $X=\left(\bigoplus_{n \in \mathbb{Z}} D^{n} M_{1}\right) \oplus Y$, where $M_{1}$ is some shift invariant subspace in $X$ and $Y$ is a reducing subspace. If $Y=\{0\}$, then the result follows. Otherwise, there is a $\psi \in L^{2}\left(\mathbb{R}^{d}\right)$ such that $\left\{D^{n} T_{k} \psi: n \in \mathbb{Z}, k \in \mathbb{Z}^{d}\right\}$ is an orthonormal basis for $Y$. Let $M_{2}=\overline{\operatorname{span}}\left\{T_{k} \psi: k \in \mathbb{Z}^{d}\right\}$ and define $M=M_{1} \oplus M_{2}$. Note that by the definition of $M_{1}$ in the proof of Proposition 1 , it follows that $D^{n} M_{1} \perp D^{m} M_{1}$ for $n, m \in \mathbb{Z}$ with $n \neq m$. So $X=\bigoplus_{n \in \mathbb{Z}} D^{n} M$ with $D^{n} M \perp D^{m} M$ when $n, m \in \mathbb{Z}$ and $n \neq m$.

2): Let $\psi$ be an orthonormal wavelet for $X$. Choose $k, l \in N$ such that $k=2^{l-1}$ and $n_{0} \in \mathbb{Z}^{d} \backslash A \mathbb{Z}^{d}$. Let $E_{1}:=\left\{\varepsilon_{0}, \varepsilon_{1}, \cdots, \varepsilon_{k-1}\right\}$ be a set of representatives of distinct cosets in $\mathbb{Z}^{d} / A^{l-1} \mathbb{Z}^{d}$. Then $F:=\left\{A^{l-1} n_{0}+\varepsilon_{0}, A^{l-1} n_{0}+\varepsilon_{1}, \cdots, A^{l-1} n_{0}+\varepsilon_{k-1}\right\} \bigcup E_{1}$ is a set of representatives of distinct cosets in $\mathbb{Z}^{d} / A^{l} \mathbb{Z}^{d}$. Indeed, for $0 \leq i, j \leq k-1$, clearly $A^{l-1} n_{0}+\varepsilon_{i}-\varepsilon_{j}=A^{l-1} n_{0} \notin A^{l} \mathbb{Z}^{d}$ if $i=j$. Now we consider the case $i \neq j$. Observe that $A^{l-1} n_{0}+\varepsilon_{i}-\varepsilon_{j} \notin A^{l} \mathbb{Z}^{d}$ equals to $n_{0}+A^{-(l-1)}\left(\varepsilon_{i}-\varepsilon_{j}\right) \notin A \mathbb{Z}^{d}$. Note that $\varepsilon_{i}-\varepsilon_{j} \notin A^{l-1} \mathbb{Z}^{d}$. So $A^{l-1}\left(\varepsilon_{i}-\varepsilon_{j}\right) \notin \mathbb{Z}^{d}$. Define two subsets $\Psi$ and $\Phi$ of $X$ and two shift invariant subspaces $P$ and $M$ as follows:

$$
\begin{gathered}
\Psi=\left\{D^{l} T_{\varepsilon_{0}} \psi, D^{l} T_{\varepsilon_{1}} \psi, \cdots, D^{l} T_{\varepsilon_{k-1}} \psi\right\}, \\
\Phi=\left\{D^{l} T_{A^{l-1} n_{0}+\varepsilon_{0}} \psi, \cdots, D^{l} T_{A^{l-1} n_{0}+\varepsilon_{k-1}} \psi\right\}, \\
P=\overline{\operatorname{span}}\left\{T_{n} f: f \in \Psi, n \in \mathbb{Z}^{d}\right\}, \\
M=\overline{\operatorname{span}}\left\{T_{n} g: g \in \Phi, n \in \mathbb{Z}^{d}\right\} .
\end{gathered}
$$

Then $\left\{T_{n} f: f \in \Psi, n \in \mathbb{Z}^{d}\right\}$ forms an orthonormal basis for $P$ due to the fact that $\psi$ is an orthonormal wavelet for $X$. The same to $M$. Define

$$
X_{1}=\bigoplus_{j \in \mathbb{Z}} D^{j} P \text { and } X_{2}=\bigoplus_{j \in \mathbb{Z}} D^{j} M
$$

Then $X=X_{1} \oplus X_{2}$. Next, we will show $X_{1}$ is a purely non-reducing affine subspace. Write

$$
Q:=\bigoplus_{j=0}^{\infty} D^{j} P
$$


Obviously $Q$ is a shift invariant subspace contained in $X_{1}$ and $P=Q \ominus D Q$. According to Proposition 2, it suffices to show that $Q$ is the maximal shift invariant subspace contained in $X_{1}$. Also by Proposition 3, it is enough to show $X_{1} \cap\left(\overline{\operatorname{span}}\left\{T_{n} L_{1}: n \in \mathbb{Z}^{d}\right\}\right)^{\perp} \subset Q$, where $L_{1}=\overline{\operatorname{span}}\left\{T_{n} X_{1}: n \in \mathbb{Z}^{d}\right\} \ominus X_{1}$. Observe that for each $j \in \mathbb{N}$,

$$
\begin{aligned}
T_{A^{j-1} n_{0}} D^{-j} \Psi & =\left\{T_{A^{j-1} n_{0}} D^{l-j} T_{\varepsilon_{0}} \psi, T_{A^{j-1} n_{0}} D^{l-j} T_{\varepsilon_{1}} \psi, \cdots, T_{A^{j-1} n_{0}} D^{l-j} T_{\varepsilon_{k-1}} \psi\right\} \\
& =\left\{D^{l-j} T_{A^{l-1} n_{0}+\varepsilon_{0}} \psi, D^{l-j} T_{A^{l-1} n_{0}+\varepsilon_{1}} \psi, \cdots, D^{l-j} T_{A^{l-1} n_{0}+\varepsilon_{k-1}} \psi\right\} \\
& \subset D^{-j} M .
\end{aligned}
$$

Then for each $j \in \mathbb{N}$,

$$
T_{A^{j-1} n_{0}} D^{-j} \Psi \subset \overline{\operatorname{span}}\left\{T_{n} X_{1}: n \in \mathbb{Z}^{d}\right\} \ominus X_{1}=L_{1}
$$

since $D^{m} M \perp X_{1}$ for all $m \in \mathbb{Z}$. Therefore, for each $j \in \mathbb{N}$,

$$
D^{-j} \Psi \subset \overline{\operatorname{span}}\left\{T_{n} L_{1}: n \in \mathbb{Z}^{d}\right\} .
$$

Hence

$$
\begin{aligned}
\bigoplus_{j \in \mathbb{N}} D^{-j} P & =\overline{\operatorname{span}}\left\{D^{-j} T_{n} D^{l} T_{\varepsilon_{0}} \psi, D^{-j} T_{n} D^{l} T_{\varepsilon_{1}} \psi, \cdots, D^{-j} T_{n} D^{l} T_{\varepsilon_{k-1}} \psi: j \in \mathbb{N}, n \in \mathbb{Z}^{d}\right\} \\
& =\overline{\operatorname{span}}\left\{D^{-j} T_{A^{-j} n} D^{l} T_{\varepsilon_{0}} \psi, D^{-j} T_{A^{-j} n_{n}} D^{l} T_{\varepsilon_{1}} \psi, \cdots, D^{-j} T_{A^{-j} n} D^{l} T_{\varepsilon_{k-1}} \psi: j \in \mathbb{N}, n \in \mathbb{Z}^{d}\right\} \\
& =\overline{\operatorname{span}}\left\{T_{n} D^{l-j} T_{\varepsilon_{0}} \psi, T_{n} D^{l-j} T_{\varepsilon_{1}} \psi, \cdots, T_{n} D^{l-j} T_{\varepsilon_{k-1}} \psi: j \in \mathbb{N}, n \in \mathbb{Z}^{d}\right\} \\
& =\overline{\operatorname{span}}\left\{T_{n} h: h \in D^{-j} \Psi, j \in \mathbb{N}, n \in \mathbb{Z}^{d}\right\} \subset \overline{\operatorname{span}}\left\{T_{n} L_{1}: n \in \mathbb{Z}^{d}\right\} .
\end{aligned}
$$

Thus $X_{1} \cap\left(\overline{\operatorname{span}}\left\{T_{n} L_{1}: n \in \mathbb{Z}^{d}\right\}\right)^{\perp} \subset \bigoplus_{j=0}^{\infty} D^{j} P=Q$. So $X_{1}$ is a purely non-reducing affine subspace. Similarly to $X_{2}$.

3): Let $X$ be a non-reducing affine subspace of $L^{2}\left(\mathbb{R}^{d}\right)$ and $X_{1}$ be the maximal reducing subspace contained in $X$. Write $X_{2}=X \ominus X_{1}$. Then $X_{2}$ is affine by Proposition 6 and $X_{2}$ is purely non-reducing since $X_{1}$ is the maximal reducing subspace in $X$. Also note that the orthogonal complement of a reducing space within another reducing space is always reducing. Then the uniqueness follows.

4): 4) follows after 2) and 3). The proof is completed.

\section{Acknowledgements}

We thank the Editor and the referee for their comments. This work is funded by the National Natural Science Foundation of China (Grant No. 11326089), the Education Department Youth Science Foundation of Jiangxi Province (Grant No. GJJ14492) and PhD Research Startup Foundation of East China Institute of Technology (Grant No. DHBK2012205).

\section{References}

[1] Weiss, G. and Wilson, E.N. (2001) The Mathematical Theory of Wavelets. In: Byrnes, J.S., Ed., Twentieth Century Harmonic Analysis-A Celebration//Proceedings of the NATO Advanced Study Institute, Kluwer Academic Publishers, Dordrecht, 329-366.

[2] Dai, X., Diao, Y., Gu, Q. and Han, D. (2002) Frame Wavelets in Subspaces of $L^{2}\left(\mathbb{R}^{d}\right)$. Proceedings of the American Mathematical Society, 130, 3259-3267. http://dx.doi.org/10.1090/S0002-9939-02-06498-5

[3] Zhou, F.Y. and Li, Y.Z. (2010) Multivariate FMRAs and FMRA Frame Wavelets for Reducing Subspaces of $L^{2}\left(\mathbb{R}^{d}\right)$. Kyoto Journal of Mathematics, 50, 83-99. http://dx.doi.org/10.1215/0023608X-2009-006

[4] Dai, X., Diao, Y. and Gu, Q. (2002) Subspaces with Normalized Tight Frame Wavelets in $\mathbb{R}$. Proceedings of the 
American Mathematical Society, 130, 1661-1667. http://dx.doi.org/10.1090/S0002-9939-01-06257-8

[5] Dai, X., Diao, Y., Gu, Q. and Han, D. (2003) The Existence of Subspace Wavelet Sets. Journal of Computational and Applied Mathematics, 155, 83-90. http://dx.doi.org/10.1016/S0377-0427(02)00893-2

[6] Lian, Q.F. and Li, Y.Z. (2007) Reducing Subspace Frame Multiresolution Analysis and Frame Wavelets. Communications on Pure and Applied Analysis, 6, 741-756. http://dx.doi.org/10.3934/cpaa.2007.6.741

[7] Li, Y.Z. and Zhou, F.Y. (2010) Affine and Quasi-Affine Dual Frames in Reducing Subspaces of $L^{2}\left(\mathbb{R}^{d}\right)$. Acta Mathematica Sinica (Chinese Edition), 53, 551-562.

[8] Gu, Q. and Han, D. (2009) Wavelet Frames for (Not Necessarily Reducing) Affine Subspaces. Applied and Computational Harmonic Analysis, 27, 47-54. http://dx.doi.org/10.1016/j.acha.2008.10.006

[9] Gu, Q. and Han, D. (2011) Wavelet Frames for (Not Necessarily Reducing) Affine Subspaces II: The Structure of Affine Subspaces. Journal of Functional Analysis, 260, 1615-1636. http://dx.doi.org/10.1016/j.jfa.2010.12.020

[10] Zhou, F.Y. and Li, Y.Z. (2013) A Note on Wavelet Frames for Affine Subspaces of $L^{2}\left(\mathbb{R}^{d}\right)$. Acta Mathematica Sinica (Chinese Edition), 33A, 89-97. 
Scientific Research Publishing (SCIRP) is one of the largest Open Access journal publishers. It is currently publishing more than 200 open access, online, peer-reviewed journals covering a wide range of academic disciplines. SCIRP serves the worldwide academic communities and contributes to the progress and application of science with its publication.

Other selected journals from SCIRP are listed as below. Submit your manuscript to us via either submit@scirp.org or Online Submission Portal.
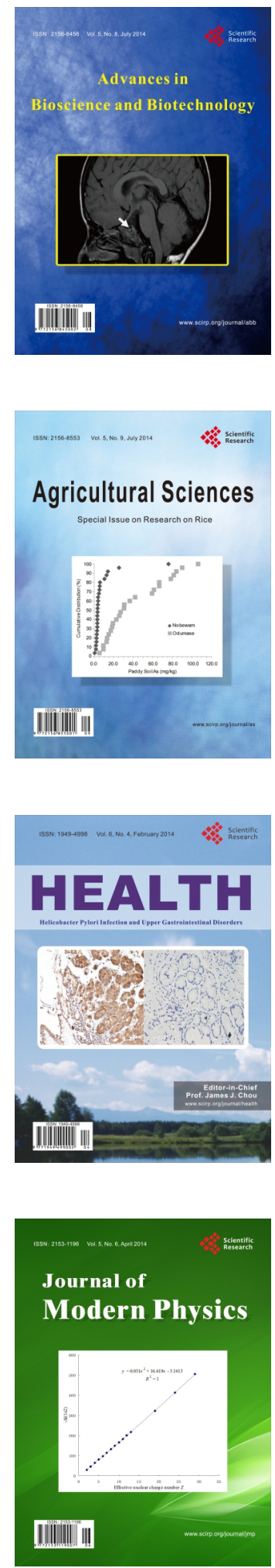
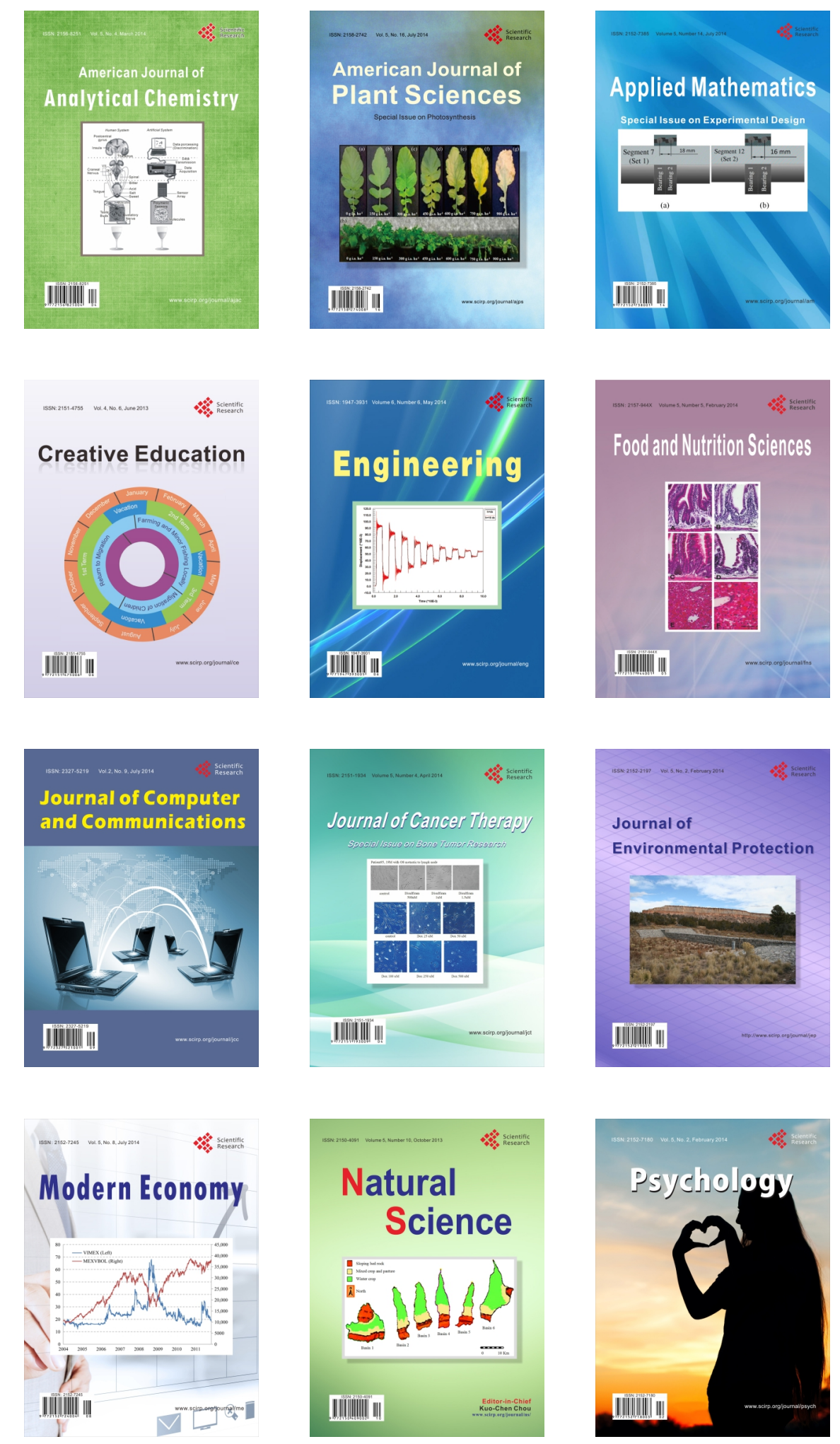